\title{
Jean-Guy Vaillancourt
}

sociologue, département de sociologie, Université de Montréal

\section{1}

\section{"Évolution, diversité et spécificité des associations écologiques québécoises: de la contre-culture et du conservationnisme à l'environnementalisme et à l'écosocialisme"}

\author{
Un document produit en version numérique par Jean-Marie Tremblay, bénévole, \\ professeur de sociologie au Cégep de Chicoutimi \\ Courriel: imt_sociologue@videotron.ca \\ Site web pédagogique : http://www.uqac.ca/jmt-sociologue/ \\ Dans le cadre de la collection: "Les classiques des sciences sociales" \\ Site web: http://www.uqac.ca/Classiques des_sciences sociales \\ Une collection développée en collaboration avec la Bibliothèque \\ Paul-Émile-Boulet de l'Université du Québec à Chicoutimi \\ Site web: http://bibliotheque.uqac.uquebec.ca/index.htm
}


Cette édition électronique a été réalisée par Jean-Marie Tremblay, bénévole, professeur de sociologie au Cégep de Chicoutimi à partir de :

Jean-Guy Vaillancourt,

\section{"Évolution, diversité et spécificité des associations écologiques québécoises: de la contre-culture et du conservationnisme à l'environnementalisme et à l'écosocialisme."}

Un article publié dans la revue Sociologie et sociétés, vol. 13, no 1, avril 1981, pp. 81-98. Montréal: Les Presses de l'Université de Montréal. [Autorisation de diffuser accordée par l'auteur le 9 mars 2004]

M. Jean-Guy Vaillancourt est sociologue au département de sociologie de l’Université de Montréal.

[Autorisation accordée par M. Jean-Guy Vaillancourt le 9 mars 2004]

8 Courriel : jean.guy.vaillancourt@umontreal.ca

Polices de caractères utilisée :

Pour le texte: Times, 12 points.

Pour les citations : Times 10 points.

Pour les notes de bas de page : Times, 10 points.

Édition électronique réalisée avec le traitement de textes Microsoft Word 2001 pour Macintosh.

Mise en page sur papier format

LETTRE (US letter), 8.5'” x 11"')

Édition numérique réalisée le 17 octobre 2004 à Chicoutimi, Ville de Saguenay, province de Québec, Canada. 


\title{
Table des matières
}

\author{
Introduction
}

1. Origine et développement des associations écologiques québécoises

2. Unité et diversité du mouvement écologique québécois

3. Spécificité et originalité des associations écologiques québécoises

\section{Résumé}




\section{Introduction}

$\underline{\text { Retour à la table des matières }}$

Il existe fort peu de descriptions et d'analyses sociologiques des associations écologiques québécoises. À part quelques mémoires de maîtrise et divers rapports financés par des organismes gouvernementaux, qui traitent d'aspects restreints du sujet ou qui sont des répertoires bien plus que des études sociologiques, et à part quelques articles épars dans des revues et des journaux qui abordent tangentiellement ces sujets, il n'y a presque rien de publié, et très peu de choses d'écrites ${ }^{1}$. Et pourtant, les écologistes québécois sont très actifs et très visibles, et ils s'expriment beaucoup depuis quelques années ${ }^{2}$. Aussi, plusieurs spécialistes des sciences sociales considèrent-ils que le mouvement écologique québécois, surtout son aile la plus radicale, représente un phénomène de plus en plus important au Québec. Par exemple, le dernier chapitre

1 Voir, par exemple, le mémoire de Louise Boileau, Étude de deux mouvements écologiques: S.V.P. et S.T.O.P., mémoire de M.Sc. en sociologie, Département de sociologie, Université de Montréal, 1975, ainsi que le Citoyen protège son environnement, publié en septembre 1974 par la Société pour vaincre la pollution (101 pages) et mes trois brefs articles : «Lettre à un militant antinucléaire », Possibles, vol. 2, n 4, été 1978, pp. 99-110, «The Ecology Manifesto and the Growth of the Movement in Quebec », Our Generation, vol. 13, n 4, automne 1979, pp. 5-7 et « Le débat public sur l'énergie aura-til lieu ? », Possibles, vol. 5, n 3, 1981.

2 En plus de ce qui s'écrit dans des journaux et des revues qui s'intéressent à l'occasion aux thèmes environnementaux (v.g. le Devoir, la Presse, le Soleil, Perspectives, Québec Science, le Temps fou, Focus Possibles, Our Generation), il existe au moins une douzaine de publications spécifiquement écologiques ayant un bon tirage au Québec, dont les plus importants sont sans doute le journal l'Environnement de la Société pour vaincre la pollution (S.V.P.). De toute urgence du Conseil québécois de l'environnement (C.Q.E.), Forêt conservation publié par les Clubs 4-H et l'Association forestière québécoise, Vers une ville nouvelle du Monde à bicyclette (M.A.B.), Transitions du Regroupement pour la surveillance du nucléaire (RSN), le Noyau publié par une commune en Gaspésie, Informations, des Amis(e) de la Terre de Québec, et la toute nouvelle revue Biosphère, publiée par le Biocreux. 
du livre la Conjoncture au Québec au début des années 80, écrit par quatre militants socialistes qui sont aussi des spécialistes des sciences sociales, se termine par une section sur le Front écologique qui clôt sur les interrogations suivantes :

Mais en fait, les revendications écologiques ne posent-elles pas un problème gigantesque au capitalisme, particulièrement dans la conjoncture de crise structurelle actuelle du système (modernisation, dépenses, recyclage, etc.) ? Jusqu'à quel point la problématique écologique ne constitue-t-elle pas ces années-ci un front privilégié pour affaiblir le capitalisme? D'autre part, en jonction avec des organisations inscrites dans une lutte sociale plus globale (syndicale et politique), le mouvement écologique ne pourrait-il pas apporter aux militants syndicaux et politiques une vision nouvelle plus large de la société à construire ? Une société dans laquelle la conservation de la vie, la qualité de la vie ne seraient pas des expressions creuses, une société critique face aux productivisme, à l'idéologie de la croissance ${ }^{3}$.

Du point de vue descriptif, une des choses les plus intéressantes que je connaisse sur l'aile progressiste du mouvement écologique québécois est la série de treize cahiers de transcriptions d'une série d'émissions radiophoniques présentées à Radio-Canada durant l'été de l'année 1980 par le réalisateur Denis Boucher, avec l'aide de l'écologiste André Delisle ${ }^{4}$. Ces treize émissions ont exploré le monde des écologistes québécois et tenté de décrire certaines de leurs expériences et de leurs réalisations dans les domaines suivants: les communautés rurales, les énergies douces, l'agriculture biologique, le recyclage, les coopératives alimentaires, les entreprises communautaires, les médias, l'autoconstruction et l'artisanat, l'alimentation naturelle, le mouvement écologique, les lieux d'échanges, les initiatives de sensibilisation, et l'écosociété. Ces 173 pages d'interviews et de commentaires nous livrent une image plutôt fidèle de ce qu'il y a de plus dynamique parmi les associations écologiques québécoises actuelles. Des militants écologistes bien connus comme Michel Bélair (coauteur du Répertoire québécois des outils planétaires ${ }^{5}$ ), François Tanguay (cofondateur de la nouvelle revue Biosphère), Clément Boulanger (du Mouvement d'agriculture biologique), Michel Jurdant (des Ami(e)s de la

3 Marielle Désy, Marc Ferland, Benoît Lévesque et Yves Vaillancourt, la Conjoncture au Québec au début des années 80: les enjeux pour le mouvement ouvrier et populaire, Librairie socialiste de l'Est du Québec, Rimouski, 1980, p. 185. De même, la dernière phrase de l'introduction de l'excellente anthologie de Boismenu, Mailhot et Rouillard sur l'histoire récente du Québec, constitue une semblable mise en lumière de l'importance des associations écologiques québécoises. En effet, les auteurs, qui sont de jeunes historiens très au fait de l'évolution actuelle du Québec, y font l'affirmation suivante : "Malgré l'étonnante force de récupération du contexte nord-américain, certaines expériences d'autosuffisance communautaire, l'établissement d'un réseau de regroupements coopératifs d'alimentation et le développement d'un mouvement anti-nucléaire constituent certes le fondement d'une critique de la production et de la morale dominantes » (Gérard Boismenu, Laurent Mailhot et Jacques Rouillard, le Québec en textes, Montréal, Boréal Express, 1980, p. 24).

4 L'Écologie, espace de vie, Service des transcriptions et dérivés de la radio, Montréal, Maison de Radio-Canada, 1980.

5 Christian Allegre et al., le Répertoire québécois des outils planétaires, vol. I, Montréal, Les Éditions alternatives Ltée, 1977. 
Terre de Québec, auteur du livre les Insolences d'un écologiste ${ }^{6}$ ), Thérèse Dumesnil (journaliste écologiste à Perspectives et auteur d'un livre tout récent sur Pierre Dansereau), Christian Ouellette (du Groupe de ressources en autoconstruction et en écologie), Michel Belleau (des Ami(e)s de la Terre de Québec), Roger Julien (de l'Alliance Tournesol) et Pierre Lacombe (de la S.V.P.) principal organisateur du Front commun pour un débat public sur l'énergie entre autres, y sont interviewés sur leurs expériences et leurs opinions, de telle sorte que l'ensemble des témoignages rapportés constitue un document indispensable pour connaître l'évolution récente et la diversité actuelle du mouvement écologique québécois.

L'intérêt grandissant pour les associations écologiques est encore trop récent pour avoir donné lieu à des études approfondies à leur sujet, mais d'ici quelques années, grâce aux recherches en cours et aux thèses et mémoires qui sont en train d'être rédigés, l'on peut prévoir qu'il y en aura, surtout si beaucoup de travaux sont réalisés sur des groupes particuliers et sur des aspects bien délimités de la question. En attendant, je crois qu'il peut être utile, à partir des études que je poursuis sur le mouvement écologique québécois au moyen d'interviews, d'observation participante et d'une analyse de la production intellectuelle et de l'action de ce mouvement, de donner ici une idée d'ensemble de son origine et de son développement, ainsi que de sa configuration actuelle et de sa spécificité par rapport au mouvement écologique dans d'autres pays comme la France et les États-Unis.

\section{Origine et développement des associations écologiques québécoises}

\section{$\underline{\text { Retour à la table des matières }}$}

La prise de conscience de l'importance de la conservation et de la nécessité de lutter contre les nuisances et contre la pollution de l'air et de l'eau n'a pas débuté brusquement au Québec au cours des années soixante, comme certains sont parfois portés à le croire. Nous avons eu au Québec l'équivalent de l'action des pêcheurs à la ligne et des conservationnistes qui sont respectivement à l'origine du mouvement écologique en France et aux États-Unis ${ }^{7}$.

6 Michel Jurdant, les Insolences d'un écologiste. Édition revue et augmentée. Montréal, Boréal Express, 1981.

7 Claude Fischer, «Le mouvement écologique et ses contradictions », Preuves, ${ }^{\circ} 19$, automne 1974, pp. 6-21. C.M. Vadrot, «Les mouvements écologiques », dans J.-P. Charbonneau et al., Encyclopédie de l'écologie, Paris, Larousse, 1977, p. 454. D.E. Morrisson, K.E. Hornback and W.K. Warner, «The Environmental Movement: Some Preliminary Observations and Predictions », dans W. Burch, N. Cheek and L. Taylor 
Songeons, par exemple, au Cercle des Jeunes Naturalistes, aux sociétés de protection de la faune, aux groupes de randonnée dans la nature, aux Clubs 4$\mathrm{H}$, à l'Association forestière québécoise, aux gens qui gravitent autour du Jardin et de l'Institut botanique de Montréal, à certaines associations savantes comme la Société de géographie de Québec, fondée en 1878, la Société linnéenne de Québec, la Société canadienne d'histoire naturelle et d'autres sociétés membres de l'A.C.F.A.S., au rayonnement considérable du Frère Marie-Victorin, puis de Pierre Dansereau, le premier véritable écologiste québécois, dont la renommée est internationale et dont l'influence a commencé à se faire sentir au Québec durant les années cinquante ${ }^{8}$. Ici donc, comme ailleurs, on trouve les précurseurs du mouvement écologique actuel chez les fervents de la nature et chez les spécialistes de la botanique et de la biologie. De plus, à la fin des années cinquante, il existait déjà plusieurs associations de riverains de lacs désireux de conserver leur milieu dans son état naturel, et des groupes de scientifiques et d'étudiants qui s'insurgeaient contre les dangers des explosions nucléaires pour des raisons de santé et de protection de l'environnement. Les comités de citoyens mis sur pied à partir de 1963 ont été eux aussi des précurseurs des groupes écologiques, étant donné leur préoccupation avec les problèmes de logement et de santé. De même, certaines associations de protection des consommateurs font figure de précurseurs du mouvement écologique.

Le premier règlement adopté au Canada contre la pollution de l'air fut voté en 1872 à Montréal. En 1931, la ville de Montréal créa une division d'inspection pour l'élimination de la fumée, à l'intérieur du Service des travaux publics et, en 1950, le Conseil municipal vota un règlement plus général contre la pollution de l'atmosphère. En 1966, la ville adopta un nouveau règlement plus sévère qui amena la fermeture de 1800 incinérateurs privés ${ }^{9}$. C'est donc dire que les pressions des citoyens contre la pollution de l'air, qui se manifestent aujourd'hui dans la lutte pour fermer l'incinérateur Des Carrières à Montréal ne date pas d'hier. Sur le plan provincial, le gouvernement du Québec mit sur pied en 1958 un Comité de pollution des eaux, qui fut remplacé en 1961 par la Régie d'épuration des eaux. À partir de 1966, le gouvernement organisa une Division du génie sanitaire sous la direction de Tony Lesauteur. Là encore la pression des citoyens y est pour quelque chose, bien qu'alors il n'existait pas encore de véritable groupe écologique se définissant comme tel.

(édit.), Social Behavior, Natural Resources and the Environment, New York, Harper and Row, 1972, pp. 259-279. Caroll Pursell, From Conversation to Ecology. The Development of Environmental Concern, New York, Thomas Crowell, 1973, Allan Schnaiberg, The Environment, From Surplus to Scarcity, New York, Oxford University Press, 1980.

8 Le livre de Thérèse Dumesnil, Pierre Dansereau, l'écologiste aux pieds nus, Montréal, Nouvelle, Optique, 1981, constitue une excellente introduction à l'œuvre de Dansereau. De son Biogeography (New York, Ronald Press, 1957) à la série de films éducatifs qu'il est en train de faire sur l'écologie Pour le ministère de l'Environnement du Québec, ce dernier n'a pas cessé de jouer un rôle clef dans le développement de l'écologie au Québec.

9 L'Assainissement de l'air dans l'île de Montréal, 1872-1980, Montréal, Communauté urbaine de Montréal, Service de l'assainissement de l'air et de l'inspection des aliments, 1980, pp. 28-30. 
Comme aux États-Unis et en France, c'est surtout à partir de 1968 qu'on passe d'un conservationnisme très modéré et peu organisé à un environnementalisme réformateur porté par des groupes plus revendicateurs. Le mouvement écologique démarre vraiment en 1970 avec la publication du premier et unique numéro de la revue Écologie-Québec par des gens faisant partie du Centre d'information sur la pollution de l'environnement, avec la création de la S.V.P. et de son équivalent anglophone S.T.O.P. (Society to Overcome Pollution), avec la fondation de Survivre (une association de scientifiques pour la survie de l'homme et de la vie), des Citoyens pour la Responsabilité Sociale dans la Science (pour lutter contre la pollution de la Union Carbide à Beauharnois), et du Conseil québécois pour l'environnement ${ }^{10}$. En 1971, c'est le groupe Sauvons Montréal, puis l'Association des Espaces Verts qui sont fondés. Dans presque tous ces groupes, dans lesquels plusieurs universitaires sont impliqués, l'idéologie n'est plus une espèce de conservationnisme de droite, mais plutôt un environnementalisme réformiste luttant contre la pollution et le gaspillage des ressources. Un certain militantisme commence même à poindre à l'intérieur de certains de ces groupes qui rejoignent un public de plus en plus large, surtout parmi les jeunes. Il y a aussi toute une série de petits groupes environnementalistes locaux qui naissent, et qui vivent, parfois seulement le temps d'une lutte ou d'une subvention, mais qui ont quand même, grâce aux mass média, un impact considérable dans la population et auprès des gouvernements. Durant l'été de 1970, la S.V.P. et S.T.O.P. sont responsables d'un pavillon écologique à Terre des Hommes. Avec l'arrivée au pouvoir du gouvernement libéral de Robert Bourassa (qui en décembre 1970 nomme Victor C. Goldbloom responsable de la qualité de l'environnement), et surtout après les événements d'octobre 1970, un mouvement communautaire rural à forte tendance écologique commence a se développer parmi les contre-culturels. Mainmise (surtout après quelques années de publication), le Répertoire québécois des outils planétaires et le vaste réseau des coopératives d'alimentation naturelle, expriment assez bien cette tendance contre-culturelle orientée vers le communautarisme rural qui se développe peu à peu à côté d'une tendance contre-culturelle plus individualiste.

À partir de 1971, l'attention de la plupart des groupes environnementalistes urbains se centre sur les diverses formes de pollution de l'air et de l'eau, sur la protection du Saint-Laurent (projet «Un fleuve, un parc »), sur l'aménagement de l'espace urbain, sur les transports publics, et sur la critique de la société de consommation. Par exemple, la S.V.P. prépare en 1971 des mémoires sur la fluoration de l'eau, sur le traitement des eaux usées, et sur l'autoroute Est-Ouest. Le livre de Chaput et Lesauteur, Dossier pollution, et le numéro spécial de la revue Critère sur l'environnement expriment bien les

10 À la suite d'un symposium sur la conservation tenu à Montréal en novembre 1969, il avait été décidé de créer un Conseil québécois pour la conservation des ressources naturelles. Un an plus tard, le Conseil québécois de l'environnement était fondé à un congrès tenu au Jardin botanique de Montréal. Le C.Q.E. publie depuis dix ans une revue écologique de qualité De toute urgence, et organise des symposia sur des thèmes environnementaux. (Michel Maldague, "Le Conseil québécois de l'environnement à l'aube d'une nouvelle décennie », De toute urgence, vol. XI, $\mathrm{n}^{\circ} 3,1981$, pp. 335-341.) 
préoccupations environnementalistes de cette période ${ }^{11}$. En mars 1971, le gouvernement du Québec regroupe la Régie des eaux du Québec et la Direction générale des équipements et de l'hygiène du milieu, pour former les Services de protection de l'environnement, avec un budget de 4 millions pour 1971 , de 12 millions pour 1972 , et de 17 millions pour $1973^{12}$. C'est à la fin de 1972 que la loi sur la qualité de l'environnement est sanctionnée à Québec par le gouvernement Bourassa à la suite de pressions de la part des groupes écologiques. Ce même gouvernement qui tente de projeter une image de défenseur de l'environnement, est fortement critiqué en avril 1973 par le Comité de défense de la Baie James, pour avoir lancé le «projet du siècle » à la Baie de James dans le but de fournir l'énergie nécessaire à une usine d'enrichissement d'uranium requise pour la production d'énergie nucléaire ${ }^{13}$.

Vers la fin de 1973, avec la crise du pétrole, c'est la question de l'énergie qui passe à l'avant-scène, puis, presque simultanément, la question des ressources alimentaires. L'analyse des environnementalistes commence alors à se radicaliser et on voit naître à ce moment-là au Québec une véritable écologie politique. Avec la crise économique qui s'amorce, c'est la société capitaliste industrielle et sa logique productiviste centrée sur le profit plutôt que sur les besoins réels des gens qui est de plus en plus attaquée. Les projets de société de conservation et même d'écosociété, l'autonomie des communautés naturelles, l'antiproductivisme, la qualité de la vie et les problèmes de simple survie sont des questions qui prennent une importance centrale. La S.V.P., avec son journal l'Environnement et ses divers comités, s'attaque à ces nouveaux problèmes, tout en continuant à lutter contre la pollution de l'air et de l'eau, et demeure un des groupes les plus dynamiques du mouvement écologique québécois. En 1974, deux groupes voient le jour, le Mouvement pour l'agriculture biologique, et les Ami(e)s de la Terre de Québec, qui expriment bien les nouvelles préoccupations. Cette même année, il y a aussi un premier effort de rassemblement des divers groupes écologiques québécois, avec l'organisation d'une Semaine de l'environnement, qui est suivie de la fondation du Regroupement québécois pour l'environnement (R.Q.P.E.). Ce regroupement réunit un ensemble hétéroclite des fervents de la nature d'une part, d'environnementalistes et de militants écologiques d'autre part, ce qui rend son existence assez précaire dès le début.

En 1975, la question nucléaire commence aussi à devenir une préoccupation majeure. Le Regroupement pour la surveillance du nucléaire est formé à Montréal en juillet 1975, pour répondre à ce problème. Le R.S.N. est un groupe pan-canadien, qui tire ses origines des inquiétudes concernant la prolifération des armes atomiques suite à l'explosion par l'Inde d'une bombe

11 Marcel Chaput et Tony Lesauteur, Dossier pollution, Montréal, Éditions du Jour, 1971 Critère, $\mathrm{n}^{\circ} 5$, janvier 1972 .

12 De $\$ 17$ millions qu'il était encore en 1976, ce budget augmente à $\$ 71$ millions en 1980, le nombre de fonctionnaires est passé de 540 à 1200 , tandis que le nombre de poursuites annuelles contre les pollueurs est passé de 100 environ à près de 800 durant ces mêmes quatre années.

13 Ce groupe avait publié peu de temps après sa fondation le livre la Baie James, c'est grave, grave, grave.... Montréal, Éditions québécoises, 1972. 
atomique créée grâce à l'achat d'une centrale CANDU par ce pays. Le R.S.N. regroupe au début environ 45 associations de toutes les régions du Canada. Maintenant, ce nombre s'élève à plus de 200 groupes. Au plan local, le Comité de protection de l'environnement de Sainte-Croix de Lotbinière est créé en 1975 pour empêcher la construction d'une centrale nucléaire sur le territoire de cette municipalité. Le Comité énergie de la S.V.P., grâce surtout à sa brochure très bien documentée sur les dangers du nucléaire civil ${ }^{14}$, prend l'initiative dans ce nouveau combat en s'opposant au nucléaire pour des raisons de sécurité, et à cause des coûts économiques et sociaux élevés de cette option. En mai 1976, il y a un nouvel effort pour relancer le R.Q.P.E., avec les États généraux de l'environnement. De nombreux groupes sont présents à la rencontre, mais la tentative de regroupement échoue finalement, non par manque d'intérêt mais parce que les groupes convoqués ont des préoccupations trop différentes les unes des autres. Chacun préfère oeuvrer seul ou avec des groupes semblables au plan idéologique. Les groupés ne semblent pas intéressés non plus à perdre une partie de leur autonomie au profit d'une fédération ou d'un regroupement.

De 1970 à 1976, il y a donc un nombre imposant de groupes environnementalistes œuvrant au Québec qui, malgré la faiblesse de leurs moyens et la précarité de leur existence, sont très actifs et très dynamiques, grâce à l'engagement de leurs membres et surtout de leurs dirigeants, à cause aussi de la prise de conscience croissante d'une bonne partie de la population québécoise au sujet des problèmes environnementaux. Ces groupes sont surtout formés de jeunes gens ayant une orientation politique de gauche libertaire. Ils arrivent parfois à exister et à subsister grâce au bénévolat et aux contributions des membres, et avec l'aide de subventions gouvernementales pour des projets de conservation d'énergie ou d'éducation écologique ${ }^{15}$.

L'année 1977 a été l'année du grand redémarrage du mouvement écologique québécois. Ce nouvel essor est dû en bonne partie à l'influence de la conjoncture internationale dans le domaine de l'énergie. L'Hydro-Québec avait alors des plans grandioses qui prévoyaient la construction de 35 centrales nucléaires au Québec. Lors de la Commission parlementaire des ressources naturelles sur l'énergie en février 1977, plusieurs groupes présentent des mémoires attaquant l'option pro-nucléaire du gouvernement. Le Regroupement pour la surveillance du nucléaire, dans un document préparé par Dorothy Henaut, Dorothy Rosenberg et Gordon Edwards, demande que le gouvernement ne construise pas Gentilly III, à cause des problèmes techniques, économiques et moraux impliqués dans cette option, et dont certains, disentils, « apparaissent tout à fait insolubles ». Ce groupe affirme que le Québec n'a pas besoin d'énergie nucléaire, puisque la demande réelle en énergie diminue et que le Québec possède d'abondantes réserves hydroélectriques qui, avec

14 Tout ce que vous aimeriez ne pas savoir sur l'énergie nucléaire et que l'on ne voudrait pas non plus vous dire, Montréal, S.V.P., 1974, 70 pp.

15 Le dossier le Citoyen protège son environnement, par exemple, qui fut produit par la S.V.P., est le fruit d'un tel projet: il constitue un répertoire de plus d'une centaine de groupes et d'actions environnementalistes ayant vu le jour au Québec avant l'été de 1974. 
une stratégie rationnelle de conservation et d'exploitation de sources d'énergie renouvelables, pourront satisfaire les besoins québécois en électricité pour bien au-delà de 50 ans. Dans son mémoire à la même Commission parlementaire, la S.V.P. insiste sur les dangers et les coûts du nucléaire, et sur les avantages de la conservation et du recyclage, de l'utilisation des ressources renouvelables et des technologies douces, de la recherche, de la décentralisation et de l'autosuffisance énergétique. La S.V.P. demande aussi un moratoire sur l'implantation de centrales nucléaires au Québec, et lance l'idée d'un vaste débat public sur l'énergie. Parmi les nombreux autres groupes et individus qui présentent des mémoires, celui qui semble avoir eu le plus d'influence sur le gouvernement des probablement le Conseil québécois de l'environnement, puisque c'est dans une perspective très proche de celle de sa demande de moratoire sur l'électronucléaire que Québec a par la suite mis de l'avant son propre projet de moratoire.

\section{Unité et diversité du mouvement écologique québécois}

$\underline{\text { Retour à la table des matières }}$

Après cette Commission parlementaire de février 1977, un besoin nouveau de regroupement antinucléaire se fait sentir parmi les groupes écologiques plus radicaux. Suite à des rencontres qui ont lieu durant l'été, un Front commun antinucléaire regroupant une quinzaine d'organismes québécois est lancé simultanément, le 20 septembre 1977, à Montréal, à Alma et à TroisRivières. On y retrouve la S.V.P., le Projet banque d'information, ContactConservation, le Groupe écologique Maisonneuve, le Monde à bicyclette, S.T.O.P., le Mouvement pour l'agriculture biologique, Sauvons Montréal, et plusieurs groupes locaux comme les Protons de Trois-Rivières, les comités de protection de l'environnement de Grondines et de Sainte-Croix de Lotbinière, la Librairie coopérative d'Alma, etc. Ces groupes organisent une manifestation antinucléaire, la première au Québec, à Gentilly le 22 octobre 1977, et environ 800 personnes y participent. Après le succès de cet événement, une cinquantaine de personnes du F.C.A.N. se réunissent, le 19 novembre, pour organiser la poursuite de la lutte antinucléaire. Elles forment le Comité écologique antinucléaire, et mettent en marche un processus pour créer une coalition antinucléaire québécoise permanente et pour tenter de mettre sur pied un nouveau regroupement écologique québécois plus dynamique, plus militant et plus souple que celui qui existait toujours, mais qui ne réussissait pas à vraiment démarrer. Ce Comité écologique antinucléaire publie au début de 
1978 une lettre manifeste aux 6 millions de Québécois. Celle-ci reprend les thèmes soulevés dans la déclaration de principe du 20 septembre et dans les documents de la S.V.P. et du R.S.N. à la Commission parlementaire de février, mais avec une insistance particulière sur le gaspillage des ressources et sur la nécessité de l'information antinucléaire au Québec. Ensuite, les Il et 12 février 1978, le Comité organise une session de formation à Montréal. Les cent personnes présentes décident sur place de créer l'Alliance Tournesol, un mouvement écologique antinucléaire décentralisé, basé sur des cellules locales autonomes, reliées entre elles autour de centres régionaux. L'Alliance Tournesol est depuis ce temps, grâce surtout au travail de quelques permanents bénévoles appuyés par de nombreux autres militants, la plaque tournante de l'activité antinucléaire chez les francophones du Québec. Il serait trop long d'analyser ici toute la production et toutes les actions de cette association: lettres aux élus et aux journaux, conférences de presse, manifestations, bulletin de liaison, vidéo, diaporama, programme à Radio Centre-Ville, conférences, sessions d'information, banque d'information avec livres, films, revues et documents, etc. Ce travail s'est fait de façon continue, malgré de petits problèmes d'organisation dûs en bonne partie au manque de ressources financières et à de légères différences d'idéologie et de personnalité qu'on retrouve dans beaucoup d'associations volontaires de ce genre.

En juin 1978, au Lac Saint-Joseph près de Québec, lors d'une fin de semaine mémorable, une grande rencontre de groupes et d'individus a lieu pour réaliser le second volet de la décision du 19 novembre 1977, c'est-à-dire la création d'un nouveau regroupement des écologistes québécois. Environ 180 groupes écologiques et plusieurs centaines d'individus y ont été invités pour former un vaste regroupement décentralisé et pour élaborer un manifeste écologique ${ }^{16}$. Non sans difficulté, un regroupement est effectivement créé, sur une base régionale, mais le projet de manifeste amorcé à partir d'une vaste consultation est mis de côté, faute de temps, et la rédaction finale est reportée à plus tard. Grâce en partie au travail préliminaire entrepris par Michel Jurdant et les Ami(e)s de la Terre de Québec, la section montréalaise du Regroupement produit finalement un manifeste quelque temps après. Ce manifeste est publié dans le bulletin du Mouvement pour l'agriculture biologique, et il est généralement bien accueilli dans le milieu des militants écologistes québécois en automne de l'année 1978 et en $1979{ }^{17}$.

16 C'est dans les termes suivants que les organisateurs présentaient le but de la rencontre: «Ce colloque sera les assises d'un grand Mouvement Écologique Kébécois (à baptiser), décentralisé mais unifié dont le but sera de créer une force socio-politique qui révolutionnera globalement et radicalement la société productiviste, hiérarchique et destructrice dans laquelle nous vivons, en contestant les choix et les valeurs sous-jacentes de son orientation actuelle et en proposant des alternatives d'une société écologique en harmonie avec l'écosphère de la planète Terre. Les chapitres locaux de ce mouvement pourront canaliser l'activité des aspirants soit vers les groupes écologiques déjà existants, soit vers le développement d'un chapitre local ou régional qui favorisera la polyvalence des préoccupations écologiques selon les priorités locales et/ou nationales. »

17 Voir les quatre commentaires de ce manifeste publiés dans Our Generation, vol. 13, n 4 , automne 1979, pp. 13-22, ainsi que la présentation que j'en ai faite (pp. 5-7). 
À la réunion du 20 janvier 1979 de la section montréalaise du Regroupement, les représentants des quatorze groupes présents mettent sur pied un comité de radio pour s'occuper d'un programme d'une heure par jour sur l'écologie, à Radio Centre-Ville. Les principaux groupes écologiques montréalais prennent chacun une heure par semaine ou par deux semaines pour mettre la population au courant de divers aspects de l'écologie politique. Il y a aussi un Comité du Journal, qui publie le journal Ecoville, un comité sur les alternatives pour promouvoir le recyclage et la conservation, un comité pour la fondation d'un café écologique, et un comité du 1er mai pour coordonner la participation des écologistes à la fête des travailleurs. Le Comité du manifeste, son mandat terminé, prépare un mémoire sur la santé et la sécurité des travailleurs pour la Commission parlementaire sur la loi 17, dans le but de rapprocher la lutte écologique de la lutte des travailleurs. Le regroupement fonde aussi un café écologique, la Vie douce, sur la rue Saint-Denis près de la rue Mont-Royal. L'orientation à donner à ce café est l'occasion d'une scission importante à l'intérieur du Regroupement de Montréal en 1980 entre une tendance plus conservatrice et plus contre-culturelle qui s'intéresse surtout à développer la Vie douce comme restaurant écologique, et une autre tendance plus politique et plus radicale qui aurait voulu en faire un centre actif de la lutte écologique. Les tenants de cette dernière tendance démissionnent du Regroupement de Montréal pour enfin se retrouver à la S.V.P. et au Front commun pour un débat public sur l'énergie. Les groupes plus stables et les mieux établis, comme la S.V.P., le Regroupement pour la surveillance du nucléaire, Sauvons Montréal, S.T.O.P., le Monde à bicyclette, le Mouvement pour l'agriculture biologique, et les divers groupes de récupérateurs de papier et de verre avaient déjà commencé d'ailleurs, depuis le début de 1980, à l'instigation de la S.V.P., à prendre leur distance vis-à-vis du Regroupement et à se réunir sur une base de fronts communs sur des luttes précises, dans le genre de la lutte amorcée durant l'été de 1979 sur la question du débat public sur l'énergie. Ce Front commun pour un débat public sur l'énergie regroupe maintenant près de 50 associations qui réclament du gouvernement la tenue d'un grand débat public sur l'ensemble de la question énergétique. C'est la S.V.P., la plus importante des organisations écologiques québécoises, qui est le fer de lance de ce Front commun qui constitue la principale activité des écologistes radicaux en ce début de 1981. L'Alliance tournesol y participe aussi, tout en collaborant surtout de façon étroite avec le Regroupement pour la surveillance du nucléaire, par exemple dans la préparation d'une prise de position conjointe sur le livre «Une stratégie pour la décennie 80 » de l'Hydro-Québec, prise de position qui a été publiée dans le Devoir du Il février 1981. Le Front commun pour un débat public sur l'énergie, qui a organisé un excellent colloque et une grande semaine sur l'énergie à l'Université du Québec à Montréal du 16 au 22 février 1981, a refusé d'aller présenter un mémoire à la Commission parlementaire, en invoquant la futilité d'une telle participation. En effet, cela n'aurait servi qu'à légitimer les politiques antiécologiques de l'Hydro-Québec en matière d'énergie. Les groupes faisant partie du Regroupement des récupérateurs à la source ont accepté de leur côté de participer aux journées de concertation sur la récupération et le recyclage organisées à la mi-février 1981 par le ministère de l'Environnement. Il faut 
dire que ces groupes de récupérateurs sont un peu dépendants pour leur financement et leurs opérations de la bonne volonté des divers paliers de gouvernement, ce qui a pour effet de les pousser dans une orientation conservationniste - environnementaliste et contre-culturelle communautaire plutôt que vers l'écologie politique et l'éco-socialisme. Le grand problème des associations écologiques québécoises demeure toujours celui du financement de leurs activités, et de la dépendance qui risque de découler vis-à-vis des instances gouvernementales à cause de ce problème, surtout en ce qui concerne le loyer des locaux, le salaire des permanents et les autres dépenses inhérentes à toute action auprès du public (publications, déplacements, recherches, etc.). Malgré ces difficultés, plusieurs de ces groupes ont non seulement un local et des militants bénévoles presque permanents, mais certains ont aussi un bulletin, un journal ou une revue qu'ils diffusent avec des tirages assez impressionnants.

Depuis un an environ, deux problèmes écologiques mobilisent beaucoup d'attention et suscitent la création de quelques nouveaux groupes et comités: ce sont le problème des déchets toxiques et celui des pluies acides ${ }^{18}$. Ces deux problèmes contribuent aussi à la radicalisation du mouvement écologique, car ils permettent d'identifier la grande entreprise privée comme étant principalement responsable de la pollution et de la détérioration écologique. Ils font comprendre les mécanismes par lesquels la grande entreprise refile les coûts sociaux de la production à la collectivité tout en gardant les profits pour elle. La radicalisation du mouvement écologique québécois s'est exprimée aussi tout récemment par la création par un groupe de femmes d'un courant écoféministe parmi les écologistes du Québec. Ce courant est en train de tenter d'opérer une convergence entre le mouvement féministe et le mouvement écologique. Une alliance du même type s'amorce aussi actuellement entre les écologistes et le mouvement ouvrier, à travers le Comité Environnement et pollution mis sur pied à la suite du Sommet Populaire de 1980 organisé par le Conseil central de Montréal de la CSN ${ }^{19}$, et à travers l'intérêt apporté à l'écologie par deux groupes socialistes démocratiques en voie de formation au Québec, le Comité des 100 et le Regroupement des militants socialistes. De même, le récent colloque sur l'autogestion organisé à l'Université de Montréal par la revue Possibles et le colloque organisé à l'UQAM par ceux qui ont publié le livre l'Impasse ${ }^{20}$, ont accordé une place importante au point de vue des groupes écologiques québécois. Il y a quelques années, dans de tels groupes et de tels rassemblements, il n'était question que du mouvement des travailleurs et du mouvement nationaliste, et parfois $\mathrm{du}$

18 Jean-Pierre Bonhomme, «Les citoyens s'intéressent de plus en plus à l'environnement », la Presse, 3 janvier 1981, p. A5.

19 La CSN s'intéresse depuis quelque temps à la lutte contre la pollution. Voir par exemple la brochure préparée par le syndicat des employés de Standard Chemical, en collaboration avec le CLSC Beauharnois-Valleyfield et le Service du génie industriel de la CSN, la Pollution par le mercure à Beauharnois. La Stanchem, principal pollueur, CSN, mai 1977, 30 pages.

20 Nicole Laurin-Frenette et Jean-François Léonard, l'Impasse. Enjeux et perspectives de l'après-référendum, Montréal, Éditions Nouvelle Optique, 1980. 
mouvement des femmes, alors qu'aujourd'hui, il y est beaucoup question aussi du mouvement écologique.

En somme, on peut dire qu'à côté des autres mouvements sociaux québécois, un véritable mouvement écologique est en train de se développer au Québec. Ce mouvement écologique comprend des associations conservationnistes plutôt modérées (vg. la plupart des 550 associations qui font partie de la Fédération des associations pour la protection de l'environnement des lacs (F.A.P.E.L.), des associations environnementalistes antipollution qui essaient de protéger la nature par des suggestions de réformes au plan individuel et collectif, et des associations d'écologistes politiques, comme celles dont nous avons surtout parlé ci-dessus. Depuis quelques années, j'ai réussi à recenser au-delà de cent associations québécoises importantes représentant ces trois différentes tendances idéologiques à l'intérieur du mouvement écologique. Si à ces trois tendances écologiques on ajoute une quatrième tendance (qui n'est pas vraiment écologique), le pseudo-conservationnisme et le pseudo-environnementalisme réactionnaires, et si on met ces quatre tendances, qui se situent sur le plan socio-politique, en parallèle avec quatre tendances semblables qu'on trouve au plan contre-culturel, on obtient la classification représentée au tableau 1.

Comme de raison, ce ne sont pas là huit types purs auxquels on peut identifier définitivement tous les membres d'une association, mais plutôt huit idéaltypes obtenus par le croisement d'un axe contre-culturel-sociopolitique avec un axe droite - gauche. Les types II-III-IV, et 2-3-4 peuvent être compris comme faisant partie du mouvement écologique québécois, bien que, à proprement parler, seuls IV et 4 devraient probablement être considérés comme étant des positions écosocialistes ${ }^{21}$.

21 Je n'insiste pas beaucoup sur les quatre catégories au plan contre-culturel. Comme pour les tendances au plan socio-politique, il y en a trois qui ont émergé successivement et qui sont «écologiques » : a) l'idéologie contre-culturelle de droite (II), centrée sur la bouffe, la musique, le hippisme californien, idéologie idéaliste et néo-libérale individualiste qui se développe au Québec à partir de 1967-1968, et qu'exprime très bien Mainmise au début ; b) l'idéologie contre-culturelle réformiste et utopique (III), qui commence vers 1971, mais qui devient importante surtout en 1975 et après, avec l'essor du communautarisme rural visant l'autosuffisance. Mainmise vers la fin, le réseau d'alimentation naturelle, la revue le Noyau représentent bien cette tendance ; c) l'idéologie contre-culturelle radicale ou le courant social alternatif qui s'exprime dans des revues comme le Temps fou (1978-) et Biosphère (1980-) et dans des groupes comme les deux M.A.B. Cette idéologie n'est pas très éloignée de l'écologie politique, avec cette différence que c'est une idéologie qui rejette la politique traditionnelle et les luttes pour le pouvoir. Ses tenants sont des socialistes libertaires plus près des anarchistes que des socialistes: d) enfin, comme sur le plan sociopolitique, il y a une position réactionnaire sur le plan contre-culturel. C'est l'idéologie contre-culturelle réactionnaire qu'on rencontre chez les contre-culturels d'extrême-droite, chez certains naturistes, chez ceux qui ont sombré dans divers genres de pensée magique, de sadomasochisme, d'érotismes, de culte des drogues, d'occultisme, de mysticisme mystifiant. Comme le pseudo-conservationnisme réactionnaire, cette position n'est pas vraiment écologique, mais représente plutôt une forme de pseudo-écologisme, et même, à la limite, d'écofascisme. 


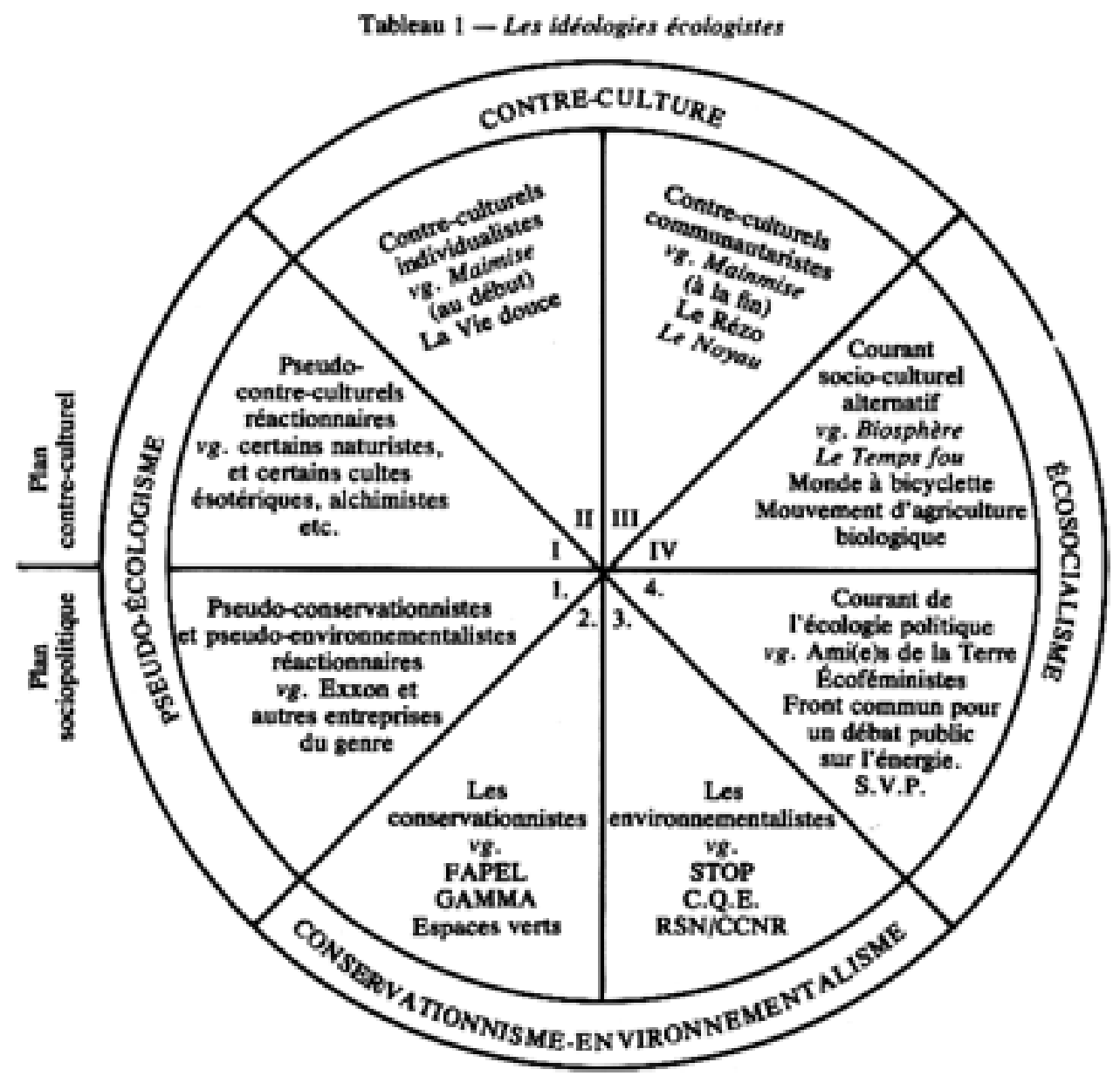

Ce que j'ai dit jusqu'ici du mouvement écologique québécois pourra sembler un peu trop descriptif et pas suffisamment analytique, même si je me hasarde à théoriser un peu avec ma classification, surtout quand je l'utilise pour tenter d'indiquer dans quelle direction le mouvement écologique et les associations qui en font partie ont évolué ces dernières années. Il est peut-être plus intéressant en sociologie de tester des hypothèses, et de pousser dans le sens de l'explication et de l'interprétation, mais dans un domaine aussi neuf et aussi peu étudié que celui-ci, il me semble qu'il faut d'abord tenter d'établir les faits avant d'échafauder des schémas explicatifs et des prédictions. J'ai préféré retracer dans ses grandes lignes le cheminement récent du mouvement écologique québécois et des principaux groupes qui en font partie, et à partir de là établir un modèle classificatoire pouvant être utile pour comprendre non seulement les grandes lignes de son développement, mais aussi sa configuration actuelle et sa diversité, puisque malgré l'émergence et l'accroissement de tendances nouvelles, les tendances plus anciennes et plus conservatrices continuent à exister et même à croître. 


\section{Spécificité et originalité des associations écologiques québécoises}

$\underline{\text { Retour à la table des matières }}$

À la lumière de tout ce que nous avons vu jusqu'ici sur les groupes écologiques québécois, je ne crois pas qu'il soit légitime de dire comme le font Julien, Lamonde et Latouche dans leur livre Québec 2001 : une société refroidie que leur action a été «très ponctuelle, sans grand impact et surtout largement symbolique $22 »$. On peut probablement dire que leur action a été « très récente », mais on ne peut certainement pas affirmer comme ils le font qu'elle a été « surtout très éphémère ${ }^{23}$ », même en ne tenant compte que de la période d'avant 1977. Dans une section de leur chapitre sur l'organisation sociétale du Québec, ces auteurs tentent de décrire le mouvement écologique québécois entre 1970 et 1976 et de prédire la dynamique de son développement futur. Mais comme les données sur lesquelles ils basent leur tentative d'explication sont insuffisantes et dans certains cas inexactes, il n'est pas surprenant que même leur description soit erronée et que leurs prédictions soient en train de s'avérer fausses. En effet, comment peut-on affirmer sérieusement que le discours des écologistes québécois est un « discours apolitique... » ou affirmer que c'est «la règle de la pensée magique et du discours incantatoire ${ }^{24}$ ? $\gg$ C'est à ce genre de conclusion qu'on aboutit quand on ne se base que sur un maigre dossier de presse et sur quelques ouvrages américains pour asseoir les faits.

Dans un texte plus long écrit à peu près à la même époque (été 1976) et qui constitue une des seules tentatives d'analyse explicative du mouvement écologique québécois, ces trois mêmes auteurs essaient d'expliquer le soidisant retard du mouvement écologique québécois et le «caractère éphémère et très récent d'une prise de conscience écologique chez les Québécois francophones 25 », sans même avoir bien réussi à démontrer qu'il y avait eu effectivement « retard» et «sous-développement» de ce mouvement. Ils invoquent toute une série de raisons pour ce « retard », par exemple, la nature

22 Pierre-André Julien, Pierre Lamonde et Daniel Latouche, Québec 2001: une société refroidie, Québec, Boréal Express, 1976, pp. 144-150.

Loc. cit.

Ibid., p. 148.

Daniel Latouche, Pierre-André Julien, Pierre Lamonde, avec la participation de Diane Poliquin-Bourassa, Perspective socioéconomique du Québec, 1re étape. Sous-système des valeurs (6). Dossier technique (6.2) : Valeurs et idéologies post-industrielles au Québec, t. II, Québec, Office de planification et de développement du Québec, 1978, p. 516. 
utilitaire et les perspectives globalisantes de l'action et de l'idéologie des associations écologiques, la grande préoccupation des Québécois avec la question nationale et avec la croissance économique, l'isolement culturel et le bas statut socio-économique et éducationnel des Québécois, le carcan législatif et le sentiment antifédéraliste qu'on trouve au Québec (le fédéral ayant, selon eux, pris l'initiative dans la lutte contre la pollution, cela pousserait les Québécois à négliger cette lutte).

Si cette analyse des raisons pour lesquelles l'action du mouvement écologique québécois serait récente, éphémère et de peu d'envergure semble bien peu convaincante, c'est sans doute parce que les faits qu'elle tente d'expliquer ne sont pas du tout établis. Ce n'est pas tellement d'un retard qu'il s'agit, à mon avis, mais d'un développement en partie différent de ce qui s'est passé ailleurs. Ce développement a été spécifique au milieu québécois, et probablement tout aussi important qu'ailleurs. Si au lieu de se demander pourquoi le mouvement écologique québécois est en retard sur le mouvement écologique américain ou français, en le mesurant à l'aune de ces derniers, on examine plutôt comment le mouvement écologique québécois est né et s'est développé, avec ses particularités propres et sa spécificité, on arrive à une toute autre conclusion. On s'aperçoit alors qu'il n'est pas possible de parler d'un «retard», et qu'il est donc oiseux d'essayer d'expliquer ce soi-disant retard, et de faire des prédictions du genre: «La société post-industrielle que nous avons décrite aux deux chapitres précédents n'a donc rien à craindre du mouvement écologique. Déjà aux États-Unis, ce dernier a été en bonne partie récupéré. Cette tendance ne pourra que s'accentuer au cours des années 1975$2001^{26}$. »

Même si les développements les plus considérables du mouvement écologique québécois ont eu lieu à partir de 1977, donc après la rédaction des deux ouvrages de nos trois futurologues, il n'en reste pas moins qu'avant cela, si on regarde attentivement, il y avait eu un développement suffisamment imposant, même si c'était forcément sous une forme légèrement différente de celle que ce mouvement a prise ailleurs, pour qu'il fut possible d'éviter des affirmations à l'emporte-pièce comme celles qu'on trouve dans ces deux écrits.

Si le mouvement écologique québécois ne s'est pas développé comme en France et aux États-Unis, malgré de grandes ressemblances venant de l'influence chez nous des écrits et des luttes des écologistes de ces pays, et de la similarité de certains problèmes écologiques d'ici et de là-bas, c'est probablement d'abord parce que les problèmes écologiques québécois sont en bonne partie spécifiques à l'environnement québécois. Les associations écologiques québécoises se sont développées en réponse à des problèmes concrets de caractère local. Si l'antinucléaire est moins développé au Québec qu'aux ÉtatsUnis et en France, c'est sans doute que l'électronucléaire y est plus développée là-bas qu'ici. C'est moins le nucléaire que toute la question de la politique énergétique qui a attiré l'attention ici, ce qui n'est pas encore tout à fait le cas

26 Julien et al., Québec 2001 : une société refroidie, p. 149. 
dans le mouvement écologique en France et aux États-Unis. De plus, notre vaste réseau de coopératives d'alimentation naturelle, qui est une des composantes importantes et même une source de recrutement pour le mouvement écologique québécois, fait l'envie de certains écologistes français, bien que les agriculteurs biologiques soient bien plus nombreux et mieux organisés là-bas qu'ici. La sensibilisation contre la pollution de l'eau, et plus récemment contre les pluies acides et même contre les déchets toxiques, semble plus grande ici qu'aux États-Unis et en France, peut-être parce que ces problèmes sont plus aigus chez nous. Et si nous avions eu dans le Saint-Laurent une marée noire de l'envergure de celles qui sont apparu à Santa Barbara ou en Bretagne, ou si nous avions eu un Three Mile Island à Gentilly, ou si le territoire du Québec était saupoudré de centrales nucléaires, peut-être aurions-nous eu nous aussi des manifestations monstres pour protester contre la pollution par le pétrole et contre l'énergie nucléaire. Le mouvement de conservation et de récupération se développe rapidement au Québec actuellement, mais c'est probablement parce que nous gaspillons plus qu'en France, et peut-être aussi parce que nos gouvernements sont plus préoccupés par ces problèmes que ne le sont le gouvernement des États-Unis et de la France. Notre climat, nos grands espaces, notre économie basée sur l'exploitation de ressources naturelles ont aussi eu une influence sur la façon dont s'est développé le mouvement écologique québécois.

Il faudrait aussi tenir compte, en examinant la spécificité du mouvement écologique québécois, du rôle particulier qu'ont pu jouer chez nous des chercheurs engagés comme Pierre Dansereau, Michel Jurdant, Michel Maldague, le groupe GAMMA, entre autres, et celui des différents paliers et agences des gouvernements, ainsi que de la relation entre chercheurs et gouvernements. En comparaison avec les États-Unis et la France, on peut dire que le gouvernement fédéral, de même que le gouvernement du Québec à partir de 1970 avec le ministre Goldbloom, ont eu une certaine préoccupation pour les questions écologiques, spécialement pour la question de la conservation de l'énergie. L'État a ainsi réussi a coopter et à drainer bien des ressources humaines dans ses programmes de changements prudents et modérés. Beaucoup des efforts qui ailleurs sont allés dans des manifestations sont récupérés ici dans des programmes gouvernementaux, et dans les mémoires, les pétitions et d'autres genres de pressions pour affecter ces programmes. Des écologistes comme Tony Lesauteur et Michel Jurdant auraient peut-être été en France ou aux États-Unis des permanents à plein temps de groupes écologiques militants, alors qu'ici ils sont allé, travailler au gouvernement pour essayer de faire passer leurs idées dans les officines du pouvoir plutôt que dans la rue et dans des contre-média. Quand l'État prend en main certaines revendications des écologistes, même en les édulcorant, le mouvement écologiste n'est pas toujours tout à fait perdant. De plus, notre système électoral, contrairement à ce qui se passe en France, ne favorise pas l'éclosion d'un parti ou de candidatures écologistes, ni l'influence politique des militants des associations écologiques. 
Par ailleurs, parce qu'il est perçu comme un mouvement social important, et à cause de son interdépendance avec les autres mouvements sociaux québécois, (le film et le livre la Fiction nucléaire illustrent bien cette interdépendance dans le cas du mouvement de libération nationale contre l'impérialisme américain), le mouvement écologique québécois a une influence considérable. Quand on retrouve la tendance d'écologie politique parmi des nationalistes, des féministes, des socialistes, des contre-culturels, des anarchistes, des libertaires, des militants étudiants, des agriculteurs, des gens qui mènent les luttes urbaines pour le logement, le transport en commun, la santé, la défense des consommateurs et la qualité de la vie en général, on hésite à déclarer comme le font les prophètes de la société refroidie: «ce n'est donc pas de ce côté que viendra une mise en question de la société postindustrielle. Tout au plus, cette préoccupation pour l'environnement servira-telle à réorienter les relations des individus avec le système et asseoir la survie de ce dernier sur des fondements différents et peut-être plus valides ${ }^{27}$. »

C'est aussi au niveau de sa production scientifique et idéologique que le mouvement écologique québécois se différencie des mouvements français et américain. Les écologistes québécois, si on les compare par exemple aux français et même aux américains, produisent moins de travaux scientifiques et en général moins d'écrits, bien que la production de petits journaux, de bulletins, de revues et de pamphlets soit impressionnante, quand on se donne la peine de la dénicher. Si l'écologie est moins visible au Québec, c'est peutêtre aussi parce que la pseudo-écologie des multinationales y est moins diffusée qu'aux États-Unis, et parce qu'à cause de la faible densité de la population et de l'abondance des ressources naturelles, les problèmes écologiques apparaissent moins pressants.

Dans les secteurs où les problèmes sont effectivement graves, par exemple ceux de la pollution des lacs, des déchets toxiques, des pluies acides, du transport urbain, de l'alimentation, de l'énergie, les associations écologiques québécoises sont très actives et elles ne donnent pas l'impression d'être en voie d'être récupérées par le système. Au contraire, la conscientisation et la radicalisation continuent de bon train, de sorte qu'il n'est plus possible de parler sérieusement, surtout depuis quelques années, d'un retard quelconque de ce mouvement par rapport à ce qui se passe ailleurs. On n'a qu'à lire les divers manifestes et les publications des différents groupes écologiques et participer un peu à leurs activités militantes ou scientifiques pour se rendre compte que ce mouvement existe bel et bien, qu'il se développe normalement, et qu'il pose les problèmes importants, comme celui des politiques énergétiques et de l'écosociété. Le mouvement écologique québécois est fortement influencé par les mouvements écologiques français et américain, mais à bien des points de vue, il n'a rien à leur envier car il est en passe de devenir un des plus importants mouvements sociaux au Québec. D'après l'observation que j'ai pu en faire, c'est un mouvement qui semble trouver de plus en plus ses appuis parmi les jeunes, les travailleurs, les femmes, les étudiants, les enseignants, les

27 Julien et al., Québec 2001 : une société refroidie, p.150 
animateurs sociaux, les techniciens, les scientifiques et de moins en moins parmi les mieux nantis, car ce mouvement a des implications très radicales au plan socio-économique et politique. Il tend à s'opposer en fait aux mêmes adversaires que le mouvement ouvrier, c'est-à-dire aux grandes entreprises multinationales et aux technocrates à leur service. Il commence à converger avec les éléments les plus ouverts dans le mouvement ouvrier et dans le mouvement socialiste, ainsi qu'avec l'aile progressiste du mouvement des femmes, du mouvement pour la démocratie de base, et du mouvement antiimpérialiste et pro-libération nationale, c'est-à-dire, en somme, qu'il est en voie de devenir un véritable mouvement d'écologie politique. Il semble bien, que c'est seulement à cette condition qu'il réussira à avoir l'impact nécessaire pour arriver à vraiment pouvoir transformer la société québécoise ${ }^{28}$. De fait, il me semble qu'aucun des mouvements que je viens de mentionner ne peut y arriver tout seul, ce qui laisse croire que les divisions et oppositions ou tout simplement l'absence d'alliances entre eux ne fait que contribuer à faciliter le retour en force du conservatisme qui est une caractéristique importante de la conjoncture actuelle. C'est donc dire que si ces mouvements continuent de sortir de leurs ghettos respectifs pour essayer de trouver les points de convergence sur lesquels ils peuvent travailler pour des revendications égalitaires, démocratiques et respectueuses de l'environnement, leur influence sera bien plus grande. Si la tendance progressiste à l'intérieur de ces mouvements s'organisait et se structurait sous forme de mouvement social et politique, ce qui ne veut pas nécessairement dire, à cause des particularités de notre système électoral, comme parti politique électoraliste, ce mouvement aurait des chances de renverser le courant conservateur actuel.

Il y a, comme nous l'avons vu, des contradictions et des différences à l'intérieur du mouvement écologique québécois, et surtout entre le mouvement écologique québécois et les autres mouvements sociaux que j'ai mentionnés. Il ne faut donc pas tout attendre du mouvement écologique, ni des autres mouvements sociaux d'ailleurs. Il faut sans doute, pour que croisse leur influence une relativisation réciproque de leurs exigences contradictoires pour les rendre compatibles entre elles, et assez de réalisme pour qu'ils soient capables de faire converger leurs luttes non seulement pour éviter le pire (c'est un départ) mais pour maximiser les chances du changement dans la direction des besoins réels de la population.

28 Le sociologue américain Charles A. Anderson affirmait il y a quelques années: « Pour être vraiment efficace, le mouvement écologique doit faire partie d'un mouvement politique établi sur une base large et orienté vers une reconstruction économique fondamentale. C'est seulement alors que l'assaut contre l'environnement pourra être renversé. » Charles A. Anderson, The Sociology of Survival, Social Problems of Growth, Homewood, III., the Dorsey Press, 1976, p. 138. 


\section{Résumé}

$\underline{\text { Retour à la table des matières }}$

Le mouvement écologique québécois s'est développé à peu, près en même temps que les mouvements équivalents en France et aux États-Unis, et selon une dynamique semblable dans ses grandes lignes. Par ailleurs, si on examine la diversité des associations qui constituent ce mouvement, on s'aperçoit qu'elles sont quand même assez différentes sous certains aspects de celles qu'on rencontre ailleurs. Cette originalité est due en bonne partie au fait que les problèmes écologiques québécois auxquels ces associations ont à faire face sont spécifiques à l'environnement québécois, et à la situation socio-économique, politique et idéologique dans laquelle elles évoluent.

\section{Summary}

The Quebec ecological movement developed at about the same time as equivalent movements in France and in the United States, and according to a fairly similar dynamic. However, if we examine the diversity of the associations making up this movement, we can see that they are nevertheless somewhat different in some aspects from those encountered elsewhere. This originality is due to a large extent to the fact that the ecological problems faced by these associations in Quebec are specific to the Quebec environment and to the socio-economic, political and ideological situation in which they are evolving.

\section{Resumen}

El movimiento ecologico quebequense se ha desarrollado casi al mismo tiempo que los movimientos equivalentes en Francia y en los Estados Unidos, y según una dinámica muy parecida en sus grandes lineas. Por otra parte si se examina la diversidad de asociaciones que constituyen ese movimiento, nos apercibiríamos que son bastante diferentes de las que se encuentran en otras partes. Esta originalidad se debe en gran parte al hecho que los problemas ecológicos quebequenses, a los cuales deben hacer frente esas asociaciones, son específicos al medio ambiente de Quebec y a la situación socioeconómica, política e ideológica en la cual evolucionan.

Fin du texte. 